\title{
Modal Mass and Length of Mode Shapes in Structural Dynamics
}

\author{
M. Aenlle $\mathbb{D}^{1},{ }^{1}$ Martin Juul $\mathbb{D}{ }^{2}$ and R. Brincker $\mathbb{D D}^{3}$ \\ ${ }^{1}$ Department of Construction and Manufacturing Engineering, University of Oviedo, Gijón 33204, Spain \\ ${ }^{2} J$ E Electronic a/s, Maserativej 3, Vejle 7100, Denmark \\ ${ }^{3}$ Department of Civil Engineering, Technical University of Denmark, Kongens Lyngby 2800, Denmark
}

Correspondence should be addressed to M. Aenlle; aenlle@uniovi.es

Received 21 February 2020; Revised 18 May 2020; Accepted 26 May 2020; Published 23 June 2020

Academic Editor: Francisco Beltran-Carbajal

Copyright (c) 2020 M. Aenlle et al. This is an open access article distributed under the Creative Commons Attribution License, which permits unrestricted use, distribution, and reproduction in any medium, provided the original work is properly cited.

\begin{abstract}
The literature about the mass associated with a certain mode, usually denoted as the modal mass, is sparse. Moreover, the units of the modal mass depend on the technique which is used to normalize the mode shapes, and its magnitude depends on the number of degrees of freedom (DOFs) which is used to discretize the model. This has led to a situation where the meaning of the modal mass and the length of the associated mode shape is not well understood. As a result, normally, both the modal mass and the length measure have no meaning as individual quantities but only when they are combined in the frequency response function. In this paper, the problems of defining the modal mass and mode shape length are discussed, and solutions are found to define the quantities in such a way that they have individual physical meaning and can be estimated in an objective way.
\end{abstract}

\section{Introduction}

The classical equation of motion for a system with $N$ degrees of freedom (DOFs) is [1-10]

$$
M \mathbf{y}(t)+C \mathbf{y}(t)+\mathbf{K y}(t)=\mathbf{p}(\mathbf{t})
$$

where $\mathbf{p}(\mathbf{t})$ is the force input vector, $\mathbf{y}(t)$ is the response vector, and $M, \mathbf{C}$, and $\mathbf{K}$ are the mass, the damping, and the stiffness matrices, respectively.

Assuming proportional damping, it is well known that the solution is given as [1-10]

$$
\mathbf{y}(t)=\Psi q(t)=\psi_{1} q_{1}(t)+\psi_{2} q_{2}(t)+\psi_{3} q_{3}(t)+\ldots
$$

where $\Psi=\left[\psi_{1} \psi_{2} \psi_{3} \ldots\right]$ is the mode shape matrix containing the real-valued mode shapes representing the spatial solution and $\mathbf{q}(\mathbf{t})$ is the modal coordinate vector containing the modal coordinates $\mathbf{q}(t)=\left\{q_{1}(t) q_{2}(t) \ldots\right\}^{T}$ representing the time solution. Inserting the solution given by equation (2) into the equation of motion, equation (1), and multiplying from the right by the mode shape matrix transpose, it can be shown that the resulting matrices are diagonalizable, and we obtain a set of equations of independent 1-DOF systems, one for each of the modal coordinates, i.e.,

$$
m_{r} \dot{q}(t)+c_{r} \dot{q}(t)+k_{r} q(t)=p_{r}(t),
$$

where $m_{r}, c_{r}, k_{r}$, and $p_{r}(t)=\psi_{r}^{T} p(t)$ are the modal mass, the modal damping, the modal stiffness, and the modal load, respectively, corresponding to the $r$-th mode.

In the frequency domain, the equation of motion is expressed as [1-10]

$$
\mathbf{H}(\omega) \cdot P(\omega)=U(\omega)
$$

where $\mathbf{H}(\omega)$ is the frequency response function matrix given by

$$
\mathbf{H}(\omega)=\left[-\omega^{2} M+i \omega C+\mathbf{K}\right]^{-1}
$$

The FRF can also be expressed in terms of modal parameters, which for proportional damped models, it is given by $[1-4]$

$$
\mathbf{H}(\omega)=\sum_{r=1}^{N_{M}} \frac{\psi_{r} \psi_{r}^{T}}{m_{r}\left(\omega_{r}^{2}-\omega^{2}+i 2 \zeta_{r} \omega \omega_{r}\right)},
$$

where $N_{M}$ is the number of modes, $\omega_{r}$ is the natural frequencies, $\zeta_{r}$ is the damping ratio, $\psi_{r}$ is the mode shape, and $m_{r}$ is the modal mass, respectively, of the $r$-th mode. 
From equations (3) and (5), it is easily seen that the modal mass is needed in all applications where the frequency response function (FRF) (or the impulse response function (IRF)) has to be constructed from the modal parameters, such as structural modification, health-monitoring applications, and damage detection $[1-4,10]$.

A mode shape is said to be mass-normalized when the modal mass is dimensionless, i.e., $m=1$. On the contrary, a mode shape is said to be unscaled if it is not mass-normalized. The mass normalized $\phi$ and the unscaled $\psi$ mode shapes are related by the following equation [1-10]:

$$
\phi=\frac{1}{\sqrt{m}} \psi,
$$

where the modal mass $m$ is a real scalar in undamped and proportionally damped models. If the mode shape $\psi$ is dimensionless, the modal mass has units of mass (kg).

The concept of modal mass is addressed in the classical books of structural dynamics $[5,6]$, modal analysis $[1-4,10]$, and also in research papers $[11,12]$. In some books of classical dynamics $[5,13]$, the concept of modal mass has also been named as generalized mass or effective modal mass.

1.1. The Concept of Effective Mass. Ewins [1] defined a new parameter denoted as effective mass which is different from the concept of modal mass. This effective mass is related to a certain mode and a certain DOF. If mass-normalized mode shapes are used, the effective mass at DOF $j$ for mode $r$ is defined as

$$
\left(m_{j j}\right)_{r}=\frac{1}{\left(\phi_{j r}\right)^{2}} .
$$

Due to the fact that mass-normalized mode shapes are unique and not subject to any arbitrary scaling factors, this new concept is also unique and represents a useful description of the behavior of the structure point by point and mode by mode [1].

In base-excited systems, the term effective mass has also been used to define a different parameter $[5,6,14]$, which must not be mistaken with the modal mass.

For a structure subjected to an acceleration support excitation, with a mass matrix $[\mathbf{M}]$, mode shapes $\{\boldsymbol{\psi}\}$, and influence coefficient $\mathbf{l}$, the modal participation factor for the $r$-th mode can be obtained as $[5,6,14]$

$$
\Gamma_{r}=\frac{\{\boldsymbol{\psi}\}_{r}^{\mathrm{T}} \cdot[\mathbf{M}] \cdot\{\mathbf{l}\}}{\{\boldsymbol{\psi}\}_{r}^{\mathrm{T}} \cdot[\mathbf{M}] \cdot\{\boldsymbol{\psi}\}_{r}}=\frac{\{\boldsymbol{\psi}\}_{r}^{\mathrm{T}} \cdot[\mathbf{M}] \cdot\{\mathbf{l}\}}{m_{r}},
$$

which provides a measure of the system mass participating in that particular mode. In engineering, the earthquake vector $\{\mathbf{l}\}$ represents the displacements of the story masses resulting from a static unit ground displacement in the direction of the seismic excitation [6].

The effective modal mass $m_{\text {effr }}$ for the $r$-th mode is defined as

$$
m_{\text {effr }}=\frac{\Gamma_{r}^{2}}{m_{r}} .
$$

The concept of effective mass was firstly proposed by Bamford et al. [14] in the early 70s, and it is based on the assumptions that the system is excited at the base and also that the base is rigid. A mode with a large effective mass is usually a significant contributor to the response of the system $m$, i.e., only the modes which have significant effective masses are needed to represent the response of the structure in a certain frequency band $[15,16]$. Moreover, the sum of the effective masses for all modes in a given response direction must equal the total mass of the structure $[5,6]$. If some modes of the system are truncated, the effective mass of the truncated modes is added directly to the base as a residual mass [15]. The equations of motion obtained for displacement and acceleration loadings are different from each other because of the construction of the effective external load $[17,18]$. When multiple constraints need to be defined by different motions, displacement loading should be the preferred one.

1.2. Modal Mass and Modal Analysis. Most of the literature related to modal mass is devoted to methodologies and techniques to estimate the modal masses of structures and mechanical systems from experiments. In [19], it is mentioned that modal mass is the least reliable parameter when classical modal analysis (CMA, also known as experimental modal analysis) is being used. Furthermore, it is also very sensitive to response magnitude.

In classical modal analysis, the modal masses are obtained from the experimental FRF using some of the several identification techniques, which are fully described in the classical books of modal analysis [1-3]. Although it is well known that a relatively high uncertainty is expected in the modal masses when using CMA, only few papers can be found in the literature discussing this subject.

Zivanovic et al. [19] estimated the modal mass corresponding to the first vertical mode of a footbridge with CMA using two different excitations (random excitation and unaveraged chirp excitation) obtaining $53.200 \mathrm{~kg}$ and $48.800 \mathrm{~kg}$, respectively, the difference being $8.3 \%$.

Allen and Sracic [20] applied CMA to determine the modal parameters in a free-free beam, excited with an impulse hammer measuring the response with a scanning laser vibrometer. The modal masses for all the modes were estimated with an error less than $20 \%$. Much of the discrepancy was attributed to the variation in the hammer blows and uncertainty in the damping estimates.

In operational modal analysis (OMA), the forces are unknown, and the modal masses cannot be estimated, i.e., only the unscaled mode shapes can be identified for each mode $[4,21]$. In order to overcome this problem, several approaches have been proposed recently. One is based on modifying the dynamic behavior of the structure by changing the stiffness and/or the mass and then performing operational modal analysis on both the original and the modified structure [21-25]. Another one is using 
the mass matrix of a finite element model [26] or considering for the experimental mode shapes having the same modal masses as those of a finite element model [27].

Several methods have also been proposed to estimate modal masses combining OMA and the response of the structure subject to a certain artificial excitation. Pavic and Brownjohn [28] developed a method for estimating the modal masses of footbridges known to respond to pedestrian excitation. Their method is based on quantifying the dynamic forces that generate the lively response, along with a characterization of the initial part of the resonant build-up due to these forces. In [29], the modal parameters of some British rock lighthouses are estimated with CMA. The most important modes have frequencies ranging between $4 \mathrm{~Hz}$ and $7 \mathrm{~Hz}$, and the modal masses are of the order of $200 t$.

Brandt et al. [30] proposed the OMAH technique where operational modal analysis is combined with monoharmonic excitation applied by an actuator. Cara [31] developed the equations needed to compute the modal masses from a state space model when CMA and OMA are combined. Guillaume et al. [32] proposed the OMAX technique, which is an operational modal analysis approach where unknown forces as well as measurable input forces, which can be deterministic or stochastic, are available.

Hwang et al. [33] proposed a method for estimating the modal masses of a structure using a mass-type damping device. Controllability and observability matrices are constructed using the identified system matrices and modal space system matrices. The modal masses are obtained considering that the product of controllability and observability matrices does not change with the type of system matrices.

Füllekrug [34] developed the equations for estimating effective masses and modal masses from base forces measured in base-driven tests. The author obtained accurate results, and the accuracy only decreases in cases where the magnitude of the effective masses is low.

1.3. Tuned Mass Dampers (TMDs). A tuned mass damper (TMD) is a dynamical device (it consists of a mass, a spring, and a damper) that is attached to a structure in order to reduce the dynamic's response of a structure [35-39]. The mass and the stiffness of the TMD are tuned to the natural frequency that needs to be damped, typically the first mode.

A TMD is characterized by three ratios: frequency ratio (natural frequency of the TMD to the natural frequency of the structure), mass ratio (mass of the TMD to the modal mass of the structure), and damping (damping of the TMD to the damping ratio of the structure) [35-37]. Thus, the modal mass of the structure plays an important role in the design of tuned mass dampers. As the mass of the damper depends on the modal mass of the structure, how the modal mass is determined is an important factor in the design of a TMD [40].

The tuning of the properties of the TMD depends on the loading acting on the structure. Den Hartog and Ormondroyd [35] proposed a method to minimize the response to sinusoidal loading. The first successful analysis of the TMD for seismic loading was introduced by Wirsching and Yao [36].
The effect of considering soil-structure interaction in the seismic response of reinforced concrete chimneys was investigated by Elias [41], where it is concluded that the increase in the mass ratio reduces the seismic response. An integrated damping system using both TMDs and double skin facade (DSF) damping system was investigated in [42]. The author concluded that this system requires a significantly reduced TMD mass ratio compared to traditional TMDs. The response of the wind turbine tower under wind loads was investigated by Gaur et al. [37]. They concluded that an optimum damping ratio exists for each mass ratio.

1.4. Mode Shape Normalization. Mode shapes can be normalized in many different ways, the most common techniques being mass normalization, normalization to the unit length of the mode shape (length scaling), and normalization to a component (usually to the largest component) equal to unity (DOF scaling). For this reason, the modal mass of a mode shape is not unique because it is directly related to the normalization method which has been used to define the mode shape [1], making the physical interpretation difficult.

Due to the fact that the modal mass corresponding to the mass-normalized mode shapes $\phi$ is $m=1$, the so-defined dimensionless modal mass introduces problems interpreting the modal equations given by equation (3) and the modal coordinates that now have the unit $[\mathrm{m} / \sqrt{\mathrm{kg}}]$. This is misleading and unfortunate.

In [1-3], the modal mass is defined as a scaling parameter for the mode shapes, i.e., it is used to convert the original unscaled mode shape vector $\{\psi\}$ to the scaled (massnormalized) mode shape vector $\{\boldsymbol{\phi}\}$. The concept of scaling factor $\alpha$, which is related to the modal mass by

$$
\alpha=\frac{1}{\sqrt{m}}
$$

is also used in the field of structural dynamics [4].

Expressions for calculating the modal mass in continuous beams can be found in the literature [5-9]. As an example, in continuous straight planar beams with length $L$, distributed mass density $\rho(x)$, and cross section with area $A(x)$, the modal mass (also denoted as generalized mass in some books of structural dynamics) corresponding to an arbitrary normalized continuous mode shape vector $\psi(\mathbf{x})$ is given by [5-9]

$$
m_{\psi}=\int_{0}^{L} \rho(x) A(x)|\psi(\mathbf{x})|^{2} \mathrm{~d} x .
$$

In discrete systems, the mode shapes are vectors, and the modal mass corresponding to the mode shape $\psi$ can be calculated with the following equation [1-8]:

$$
m=\psi^{T} \mathbf{M} \psi
$$

where $\mathbf{M}$ is the mass matrix.

A mode shape is said to be normalized to the unit length if its length is unity. In one-dimensional continuous systems, the Euclidean length $L_{E}$ of a function $\psi(x)$, also known as Euclidean norm or $L^{2}-$ norm, is defined as [43] 


$$
L_{E}=\sqrt{\int_{0}^{L}|\psi(x)|^{2} \mathrm{~d} x},
$$

where the subindex ' $E$ ' indicates Euclidean.

In discrete systems, the length of the mode shape vector $\psi$ (length of a vector in a Euclidean space) is defined as [43]

$$
L_{E}=\sqrt{\psi^{\mathrm{T}} \psi}
$$

A mode shape $\psi$ can be scaled to the unit length by

$$
\psi_{\mathbf{L}}=\frac{\psi}{L_{E}}
$$

where $\psi_{\mathrm{L}}$ indicates the mode shape normalized to the unit length (Euclidean length equals to unity).

It is easy to realize that this procedure will define the modal mass estimated from equation (13) as something that is heavily dependent upon the number of DOFs in the model. In fact, it is easy to show that, for this case, the modal mass is approximately proportional to

$$
m \propto \frac{1}{N}
$$

Another approach to scaling is to scale the largest component (or some other component) to a certain value (for instance, equal to unity) [1-7]. This introduces the DOF scaled mode shape $\left\{\psi_{D}\right\}$ :

$$
\left\{\psi_{D}\right\}=\frac{\{\psi\}}{\psi_{\text {Dn }}},
$$

where $\psi_{D n}$ is the considered DOF used for scaling.

If the mode shapes are normalized to the largest component equal to unity, the DOF used for normalization should be the same in the FE model and the test, i.e., the DOF must be shared. If this is not the case, then the modal mass is dependent upon the selected DOF.

1.5. Physical Interpretation of Modal Mass. Although the concept of modal mass is defined in the classical books of structural dynamics and modal analysis, the physical interpretation of this modal parameter is rarely addressed.

The modal mass for the undamped and the proportionally damped cases is usually defined as a scaling factor for the mode shapes $[1,3,11]$, i.e., it is used to convert the original unscaled mode shape vector $\{\psi\}$ to the more useful mass-normalized mode shape vector $\{\phi\}[1-4]$.

When using modal superposition (see equation (3)), the modal mass represents the mass of the single DOF systems, after decoupling the equations by transformation to modal coordinates [1-4].

In [44], it is demonstrated that the modal mass of a simply-supported beam, when the mode shapes are normalized to the largest component equal to unity, is the half of the total mass of the system, whereas in fixed-fixed beams, the modal mass is equal to the total mass of the system.

However, it seems like none of the classical ways of dealing with the scaling problem of the mode shapes leads to a modal mass that has the needed clarity in terms of physical meaning. Furthermore, since the results are dependent upon the number of DOFs in the model, the individual quantities do not seem to have much meaning at all.

In this paper, a new and better definition of the modal mass is presented. This definition leads to significant improvements in the physical understanding of this modal parameter. Firstly, the most important requirements for a new definition of the modal mass are proposed. Then, a new expression for the length of a continuous mode shape is formulated introducing the concept of the volume matrix, which again leads to a new definition of the modal mass for the continuous case. The expressions derived for continuous systems are later extended to discrete cases. Finally, the concepts and equations formulated in the paper are illustrated by simple examples.

\section{Basic Requirements}

In order to achieve a better understanding of modal mass, one would expect that a new definition would lead to a better physical meaning of the modal mass, for instance, that the physical unit is indeed $[\mathrm{kg}]$ and that the size of the modal mass is a measure of the amount of mass moving in a given mode.

Similarly, for the length of the mode shapes, since the mode shape is pure geometry, it is natural to expect that a good measure of the length is a pure geometrical measure, independent of the mass distribution in the considered body. It is also expected that since a "shape" normally means a continuum of dimensionless numbers, the length is dimensionless and a measure of the average movement over the mode shape.

For practical applications (and for the physical meaning), it is important that the ways to estimate modal mass and mode shape length are objective so that different people will arrive at the same number for a given mode and a given structure. At least, they should be able to agree on some simple rules for obtaining these quantities so that they independently would arrive at the same estimates.

At this point, we will conclude that the most important requirements for a new definition of the modal mass are as follows:

(i) The modal mass must be physically meaningful, having the unit of $[\mathrm{kg}]$ and being a measure of the amount of mass moving in a given mode

(ii) The length of the mode shape must be a pure geometrical quantity, describing the average movement of the considered mode

(iii) Estimation of modal mass and mode shape length must be objective so that estimates obtained by different people are equal

\section{Basic Concepts}

We will introduce basic concepts considering the formulation in the continuous case where a mode shape $\psi=\psi(\mathbf{r})$ is given as a function of the position $r=\{\mathbf{x}, \mathbf{y}, \mathbf{z}\}^{\mathrm{T}}$. The mode 
shape at a given point $r$ has the three mode shape components $\psi_{\mathrm{x}}, \psi_{\mathrm{y}}, \psi_{\mathrm{z}}$, resulting in the magnitude

$$
|\psi|=|\psi(\mathbf{r})|=\sqrt{\psi_{x}^{2}(r)+\psi_{\mathrm{y}}^{2}(r)+\psi_{z}^{2}(r)} .
$$

The modal mass for the continuous case is now naturally defined as

$$
m=\int_{V} \rho|\psi|^{2} \mathrm{~d} V
$$

where integration is performed over the volume $V$ of the considered structure and $\rho=\rho(r)$ is the mass density. Similarly, the total mass is defined as

$$
M=\int_{V} \rho \mathrm{d} V .
$$

3.1. Mode Shape Length and Modal Mass. For the continuous case, we will define the squared length $L^{2}$ of the mode shape $\psi$ as the average of the squared length $|\psi|^{2}$ of the mode shape over the considered volume $V$ :

$$
L^{2}=\frac{1}{V} \int_{V}|\psi|^{2} \mathrm{~d} V
$$

This secures that the length definition has the same unit as the mode shape. Thus, if the mode shape is dimensionless, so is the length. If the mass density $\rho$ is constant, then from equation (20), we have

$$
m=\rho V \frac{1}{V} \int_{V}|\psi|^{2} \mathrm{~d} V=M L^{2},
$$

which is the central equation of this paper stating the following for the case of constant mass density.

The modal mass is equal to the product between the total mass of the structure and the squared length.

If the considered body is constituted by two parts with two volumes, $V_{1}$ with the mass density $\rho_{1}$ and $V_{2}$ with the mass density $\rho_{2}$, then the corresponding result is

$$
m=\frac{V_{1}}{V_{1}} \int_{V_{1}} \rho_{1}\left|\psi_{V_{1}}\right|^{2} \mathrm{~d} V+\frac{V_{2}}{V_{2}} \int_{V_{2}} \rho_{2}\left|\psi_{V_{2}}\right|^{2} \mathrm{~d} V=M_{1} L_{1}^{2}+M_{2} L_{2}^{2},
$$

where $\psi_{\mathbf{V}_{2}}$ and $\psi_{\mathbf{V}_{2}}$ indicate the DOFs of the mode shape $\psi$ in volumes $V_{1}$ and $V_{2}$, respectively, and

$$
\begin{aligned}
& L_{1}^{2}=\frac{1}{V_{1}} \int_{V_{1}}\left|\psi_{V_{1}}\right|^{2} \mathrm{~d} V, \\
& L_{2}^{2}=\frac{1}{V_{2}} \int_{V_{2}}\left|\psi_{V_{2}}\right|^{2} \mathrm{~d} V
\end{aligned}
$$

are the partial lengths now defined over the partial volumes $V_{1}$ and $V_{2}$, respectively.

Equation (20) easily generalizes to cases with many volumes with constant mass density.
As indicated above, the total mass can either be calculated as the integral of the mass density over the total volume, equation (21), or as the two similar integrals over the two partial volumes $V_{1}$ and $V_{2}$ :

$$
M=\int_{V_{1}} \rho_{1} \mathrm{~d} V+\int_{V_{2}} \rho_{2} d V=M_{1}+M_{2} .
$$

3.2. Moving Mass. Now, assuming that the mode shapes vanish inside $V_{2},|\psi(\mathbf{r})|^{2}=0$, for $r \in V_{2}$, and using equation (24) with $L_{2}^{2}=0$, we get

$$
m=M_{1} L_{1}^{2},
$$

which is obviously the right answer.

Using equation (23) on the total volume, we get

$$
m=\mathbf{M} L^{2}=\frac{\mathbf{M}}{V} V L^{2}=\frac{\rho_{1} V_{1}+\rho_{2} V_{2}}{V_{1}+V_{2}} V L^{2} .
$$

Due to the fact that

$$
L^{2}=\frac{1}{V} \int_{V}|\psi|^{2} \mathrm{~d} V=\frac{1}{V}\left(\frac{V_{1}}{V_{1}} \int_{V_{1}}\left|\psi_{V_{1}}\right|^{2} \mathrm{~d} V+\frac{V_{2}}{V_{2}} \int_{V_{2}}\left|\psi_{V_{2}}\right|^{2} \mathrm{~d} V\right),
$$

the following relationship between the total length and the partial lengths is derived:

$$
L^{2} V=L_{1}^{2} V_{1}+L_{2}^{2} V_{2} .
$$

If $L_{2}^{2}=0$, equation (30) results in

$$
L^{2} V=L_{1}^{2} V_{1},
$$

and equation (28) can also be expressed as

$$
m=\frac{\rho_{1} V_{1}+\rho_{2} V_{2}}{V_{1}+V_{2}} V_{1} L_{1}^{2} .
$$

Equation (32) is equal to equation (23) only if $\rho_{1}=\rho_{2}$ or $V_{2}=0$. This illustrates the validity of equations (22)-(24).

If $\rho_{1}=\rho_{2}=\rho$, then from equation (32), we have that $m=M L^{2}=M_{1} L_{1}^{2}$, i.e., we still get the same result for the modal mass. So, adding the dead mass $M_{2}$ does not change the modal mass because $L_{2}^{2}=0$.

However, since we are looking for a unique relationship between the modal mass and the length, we want the total moving mass to be a well-defined quantity. We can find that moving mass $M_{1}$ from equation (23), by defining the mode shape $\psi_{U}$, as a unitary translation inside $V_{1}$, that is, for $|\psi(\mathbf{r})|^{2}=1, r \in V_{1}$ because then using equation (23),

$$
m=\rho V \frac{1}{V} \int_{V}\left|\psi_{\mathrm{U}}\right|^{2} \mathrm{~d} V=\rho \int_{V_{1}} \mathrm{~d} V=M_{1} .
$$

So, if the total moving mass is smaller than the total mass in the considered system, dead mass is present.

The moving mass is a well-defined quantity, and equation (23) defines a unique relation between the modal moving mass and the mode shape length. 
3.3. Apparent Mass. In the general case of a varying mass density, we can define an average mass density as

$$
\langle\rho\rangle=\frac{M}{V}=\frac{1}{V} \int_{V} \rho \mathrm{d} V
$$

so that equation (23) still holds, but where the length is now defined as

$$
L^{2}=\frac{1}{V} \int_{V}|\psi|^{2} r d V,
$$

where $r=\rho /\langle\rho\rangle$, and the total mass is given in terms of the average density $M=\langle\rho\rangle V$. We shall not use this definition of the length, the reason being that the quantity contains information about the mass distribution, and thus, as defined in equation (35), is not a pure geometrical measure.

Instead, we will start from equation (24) formulated for an arbitrary number of constant mass distributions

$$
m=\sum_{n} M_{n} L_{n}^{2}
$$

From equations (24) and (26), it is clear that the total mass is $M=\sum_{n} M_{n}$ and that the total length is

$$
L^{2}=\frac{1}{V} \sum_{n} V_{n} L_{n}^{2}
$$

so that from equation (36), we have

$$
m=M_{a} L^{2}
$$

where the apparent total mass $M_{a}$ is given by

$$
M_{a}=V \frac{\sum_{n} M_{n} L_{n}^{2}}{\sum_{n} V_{n} L_{n}^{2}} .
$$

The advantage of using the definition of the length given by equation (37) is that the length remains a pure geometrical quantity. Unfortunately, this introduces a new mass quantity, namely, the apparent total mass $M_{a}$, which for nonuniform mass distribution is different from the total mass and changes value from mode shape to mode shape.

From equation (39), it is clear that if and only if the mass density is uniform, then $M_{a}=M$. Thus, the deviation of the apparent total mass $M_{a}$ from the total mass $M$ is a measure of how much the mass distribution deviates from the uniform distribution.

3.4. Discrete Formulations. The discrete formulation follows directly from the continuous formulation replacing the integrals with proper similar summations. Similar to equation (22), the squared length $L^{2}$ of the mode shape $\psi$ over a discrete number $N$ of nodal points in the $3 \mathrm{D}$ space is then

$$
L^{2}=\frac{1}{V} \sum_{n}|\psi|_{\mathbf{n}}^{2} \Delta V_{n},
$$

where $|\psi|_{n}^{2}$ is the length of the mode shape $\psi=\psi\left(r_{n}\right)$, where the vector $\mathbf{r}_{\mathbf{n}}$ is pointing to the nodal point $n$ representing the volume $\Delta V_{n}$. Inserting the volumes $\Delta V_{n}$ into the diagonal matrix $\mathbf{V}$ and, similarly, collecting the DOF of the mode shape in the mode shape vector, equation (36) can be written as

$$
L^{2}=\frac{1}{V} \psi^{\mathrm{T}} \mathbf{V} \psi
$$

We should be aware that we are, here, using the same symbol " $\psi$ " for the mode shape as before, even though before $\psi$ was vector field in the $3 \mathrm{D}$ space but now it is actually a discrete vector containing the DOFs of the mode shape. The discrete counterpart to equation (23) is now

$$
m=\rho V \frac{1}{V} \psi^{\mathrm{T}} \mathbf{V} \psi=M L^{2},
$$

which, as before, is only valid for a constant mass density $\rho$. Comparing this equation to the classical equation for the modal mass given by equation (13) naturally extends the definition of the volume matrix to the general nondiagonal form

$$
V=\frac{M}{\rho},
$$

where $\mathbf{V}$ and $\mathbf{M}$ are the volume and mass matrices of the system, respectively. In this more general understanding of the volume matrix, the matrix can be full and can content rotational degrees of freedom. This also means that the length definition given by equation (37) naturally extends to cases with rotational degrees of freedom.

For two different volumes, $V_{1}$ with the mass density $\rho_{1}$ and $V_{2}$ with the mass density $\rho_{2}$, equation (24) still holds but now with the length definitions:

$$
\begin{aligned}
& L_{1}^{2}=\frac{1}{V_{1}} \psi_{1}^{\mathrm{T}} \mathbf{V} \psi_{1}, \\
& L_{2}^{2}=\frac{1}{V_{2}} \psi_{2}^{\mathrm{T}} \mathbf{V} \psi_{2} .
\end{aligned}
$$

For the general case of a varying mass density, we find the discrete expression for the length from equation (24):

$$
L^{2}=\frac{1}{V} \int_{V}|\psi|^{2} \rho \mathrm{d} V,
$$

which directly leads to

$$
L^{2}=\frac{1}{V} \psi^{\mathrm{T}} \mathbf{M R}^{-1} \psi .
$$

If the mass matrix $\mathbf{M}$ is diagonal, then $\mathbf{R}$ is a diagonal matrix containing the elements $\rho_{n}$ representative for the volume $\Delta V_{n}$. Since $\mathbf{M}$ is symmetric, $\mathbf{M R}^{-1}$ can be replaced by $\mathbf{R}^{-1} \mathbf{M}$ or by the symmetric form $\mathbf{R}^{-1 / 2} \mathbf{M} \mathbf{R}^{1 / 2}$. At this moment, it is interesting to note that the symmetric form $\mathbf{R}^{-1 / 2} \mathbf{M} \mathbf{R}^{1 / 2}$ naturally defines a new definition of a mass scaled mode shape $\psi_{\mathbf{m}}=\mathbf{R}^{-1 / 2} \boldsymbol{\psi}$, but since it leads to mode shapes with the unfortunate unit $1 / \sqrt{\mathrm{kg}}$, we will not follow this lead any further.

Equation (46) naturally suggests the general volume matrix: 


$$
\mathbf{V}=\mathbf{M R}^{-1},
$$

which is not necessarily diagonal. For instance, using an FE model, with the value $\rho_{n}$ of the mass density in a certain element with the local mass matrix $\mathbf{M}_{n}$, the corresponding local volume matrix $V_{n}$ is defined according to equation (43):

$$
\mathbf{V}_{\mathbf{n}}=\mathbf{M}_{\mathbf{n}} / \rho_{n}
$$

and the global volume matrix is then assembled by the same procedure as when assembling the local mass matrices to form the global mass matrix. Following this procedure, equations (32)-(35) still hold in the general case.

Before we leave this section, let us conclude that the two important scalar quantities, the total mass $M$ and the corresponding total volume $V$, can be found from any discrete converged model as

$$
\begin{gathered}
M=\psi_{\mathrm{U}}^{\mathrm{T}} \mathbf{M} \psi_{\mathrm{U}}, \\
V=\psi_{\mathrm{U}}^{\mathrm{T}} \mathbf{V} \psi_{\mathrm{U}},
\end{gathered}
$$

where $\psi_{\mathrm{U}}$ is a unitary translation vector as mentioned earlier. We could also find the total mass and total volume adding all matrix elements corresponding to translational degrees of freedom (but then, we have to take into account if 2 or 3D formulations are being used dividing the total sum by 2 or 3 , respectively).

\section{SEREP Reduction}

In this section, we will show that the so-defined modal mass and modal length are invariant to the SEREP (system equivalent reduction expansion process) reduction technique [45]. For this case, it is practical to consider a mode shape cluster $\Psi$ instead of the single mode shape. We can then define the diagonal modal mass matrix

$$
\left[m_{n}\right]=\Psi^{\mathrm{T}} \mathbf{M} \Psi .
$$

Using SEREP, we have the reduced mass matrix

$$
\mathbf{M}_{\mathbf{a}}=\mathbf{T}^{\mathrm{T}} \mathbf{M} \mathbf{T},
$$

where $\mathbf{T}=\Psi_{\mathbf{a}} \Psi_{\mathbf{a}}^{+}$(and where we assume full rank, i.e., $\Psi_{\mathbf{a}}$ is $\mathbf{N}_{\mathrm{a}} \times \mathbf{N}_{\mathbf{a}}$ ). The modal mass matrix for the reduced mass matrix is

$$
\left[m_{a n}\right]=\Psi_{\mathrm{a}}^{\mathrm{T}} \mathbf{M}_{\mathrm{a}} \boldsymbol{\Psi}_{\mathrm{a}}=\boldsymbol{\Psi}_{\mathrm{a}}^{\mathrm{T}}\left(\boldsymbol{\Psi}_{\mathrm{a}} \Psi_{\mathrm{a}}^{+}\right)^{\mathrm{T}} \mathbf{M}\left(\boldsymbol{\Psi}_{\mathrm{a}} \boldsymbol{\Psi}_{\mathrm{a}}^{+}\right) \boldsymbol{\Psi}_{\mathrm{a}} .
$$

However, since $\left(\Psi_{\mathbf{a}} \Psi_{\mathbf{a}}^{+}\right)=\mathbf{I}$, from equation (52), it is derived that

$$
\left[m_{a n}\right]=\left[m_{n}\right] .
$$

Using the volume matrix $\mathbf{V}$ from equation (41), we can define the modal length matrix

$$
\mathbf{L}^{2}=\frac{1}{V} \Psi^{\mathrm{T}} \mathbf{V} \Psi
$$

This matrix is clearly nondiagonal but positive definite so that the notation $\mathbf{L}^{2}$ makes sense. Now, defining the SEREPreduced volume matrix

$$
\mathbf{V}_{\mathbf{a}}=\mathbf{T}^{\mathrm{T}} \mathbf{V} \mathbf{T},
$$

we can calculate the length in the reduced system:

$$
\mathbf{L}_{a}^{2}=\frac{1}{V} \Psi_{\mathrm{a}}^{\mathrm{T}} \mathbf{V}_{\mathrm{a}} \boldsymbol{\Psi}_{\mathrm{a}} .
$$

And following the same procedure as for the modal mass,

$$
\mathbf{L}_{a}^{2}=\frac{1}{V} \Psi_{\mathrm{a}}^{\mathrm{T}} \mathbf{T}^{\mathrm{T}} \mathbf{V} \mathbf{T} \Psi_{\mathrm{a}}=\frac{1}{V} \Psi^{\mathrm{T}} \mathbf{V} \boldsymbol{\Psi}=\mathbf{L}^{2},
$$

or in words,

Modal mass and length matrices are invariant to SEREP.

We should note that since the modal mass and modal length are the same in all reduced systems when using SEREP [45], equation (19) still holds in all reduced systems if we use the simple rule that the total mass should be estimated from the original system. The same is true for the total volume.

This means that the two key quantities, the total mass and the total volume, have to be estimated for the considered system once and for all. One can use an experimental approach and use a weight scale to measure the total weight and a volume scale, for instance, a fluid expansion method, to measure the volume. This is of course the best possible way to estimate these two key quantities.

If we cannot use the experimental way to estimate these quantities, then we could use an analytical model or a numerical model. An analytical model is often the simplest and the best approach. For example, if we consider a beam with length $L$ and constant area $A$ made out of material with the constant mass density $\rho$, then the total mass is simply $\mathbf{M}=\rho A L$, and the total volume is simply $V=A L$.

If we want to use a finite element model to estimate the quantities, then like for all other quantities, the model must have converged in order to make sure that the estimated quantities are reliable.

\section{Mode Shape Scaling}

We have now established the important linear relation between the total mass of the considered system, the modal mass of the considered mode shape, and the length of the considered mode shape in case of uniform mass density:

$$
m=M L^{2} .
$$

And we will now consider suitable rules for how to choose the value for $L$. When a meaningful value for $L$ has been established, the modal mass is then totally defined by equation (58). As explained in Section 1, we have to at least consider the classical scaling, the length scaling, and the DOF scaling.

5.1. Length Scaling. Considering the arbitrarily scaled mode shape $\psi$ with the length $L$, then a classical length scaling of $\psi$ would be defining the scaled mode shape $\psi_{\mathrm{L}}$ as 


$$
\psi_{\mathrm{L}}=\frac{\psi}{L}
$$

Using equation (41), we find the resulting length of $\psi_{\mathrm{L}}$ as

$$
L^{2}=\frac{1}{V} \psi_{\mathbf{L}}^{\mathrm{T}} \mathbf{V} \psi_{\mathbf{L}}=\frac{1}{L^{2}} \frac{1}{V} \psi^{\mathrm{T}} \mathbf{V} \psi=1
$$

Thus, the classical length scaling leads to a version of equation (55), where

$$
m=M
$$

or in words,

Using length scaling, the modal mass in constant mass density systems is always equal to the total mass of the considered system.

One can say that this is a simple rule and at least easy to remember and to use and also practical because, now, different people can easily arrive to the same number (or at least similar estimates) because the art is only to estimate the total mass of the system.

The drawback is of course that this modal mass does not give us any information about how much mass is actually moving in the different modes. If mass density is constant, all the modes have the same modal mass. Even a local mode, where nearly all movements are localized to a very small region, still has the same modal mass. This is unfortunate, but if one can live with this limitation, it might be a good way to deal with the modal mass.

In nonconstant mass density systems, the modal mass is different for each mode and equal to the apparent mass.

5.2. DOF Scaling. The classical DOF scaling is based on choosing one of the DOFs in the system and then scale this value to a certain number, for instance, to unity [1-4].

Before we go further into this scaling, let us consider an abstract example. Like in Section 3, let us consider the case where the continuous body is constituted by two parts with two volumes, $V_{1}$ and $V_{2}$, and let us assume that we consider the abstract mode shape $\psi_{\mathrm{U}}$ as an unitary translation inside $V_{1}$ and zero inside $V_{2}$.

$$
\left|\psi_{\mathbf{U}}(\mathbf{r})\right|^{2}= \begin{cases}\mathbf{1} ; & \mathbf{r} \in \mathbf{V}_{1}, \\ \mathbf{0} ; & \mathbf{r} \in \mathbf{V}_{2}\end{cases}
$$

Just for simplicity, let us assume that the mass density is constant, so from equation (20),

$$
m=\int_{V_{1}}\left|\psi_{\mathrm{U}}\right|^{2} \rho \mathrm{d} V=\rho V_{1}=\mathbf{M}_{1} .
$$

Thus, the modal mass is then equal to the total mass inside the volume $V_{1}$. It is also clear that if $V_{1}$ extends to the total volume $V=V_{1}+V_{2}$, then the modal mass becomes equal to the total mass of the system.

This case illustrates the DOF scaling, where the maximum value of $\left|\psi_{\mathbf{U}}(\mathbf{r})\right|^{2}$ is scaled to unity. Inspired by this case, we will define the DOF scaled version as

$$
\psi_{\mathrm{D}}=\frac{\psi}{\max (|\psi(\mathbf{r})|)} .
$$

Using this scaling, the modal mass has the properties

$$
m \leq M \text {, }
$$

and thus is a direct measure of how much mass is moving in the considered mode shape. In words, we can express this as

Using largest component equal to unity, the modal mass in constant mass density systems is always smaller than or equal to the total mass of the considered system.

The advantage of this scaling is just that the size of the modal mass now makes totally sense, but the drawback is that we need to scale the largest component to unity, which is the same as scaling:

$$
\max \left|\psi_{D}\right|=1
$$

In a model with only a few components, where one of the DOFs is not directly equal to the DOF where the mode shape is maximum, it is not so easy to find the scaling. The solution is to know the ratio between one of the DOFs of the model and the value $\max \left|\psi_{\mathrm{D}}\right|$ found in a refined model (ideally, a model where all quantities of importance have converged).

5.3. Scaling Using SEREP. We start out with an initial model where all important quantities have converged towards a stable value; then, it is easy to conclude from the earlier analysis that, for the length scaling, all reduced models satisfy equation (61), i.e., in all reduced models, the modal masses are the same and equal to the total mass of the system.

Using DOF scaling, all common DOFs are still the same, and the length, which can be estimated in any of the systems reduced from the initial system, is the same; so, also, all modal masses are the same, and the property expressed in equation (65) is true in all the reduced systems. One should note that equation (66) only holds in systems which are reduced to a size where $\max |\psi|$ is the same as in the initial system. If this is not the case, then $\max |\psi|$ must be estimated in a more refined system.

\section{Examples}

6.1. A Cantilever Beam with Constant Density. Several simulations were carried out on a planar finite element model of a cantilever beam with constant mass density and constant cross section (Figure 1). The geometric and material properties of the cross section are also shown in Figure 1.

The analytical mode shapes corresponding to the first three modes and normalized to the largest component equal to unity are shown in Figure 2 (solid lines). On the contrary, the analytical modal masses and the squared length of the mode shapes are presented in Table 1. In this particular case, the largest component is at the free border of the beam for all 


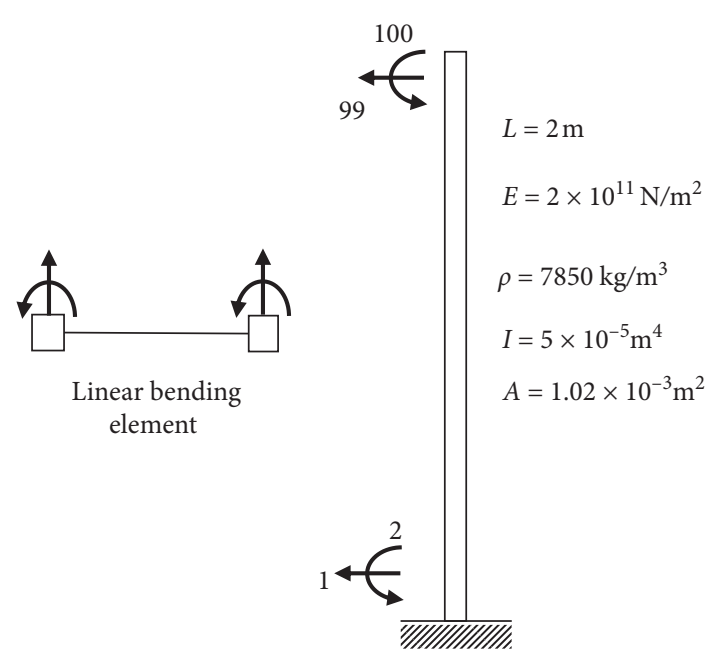

Figure 1: Cantilever beam with constant mass density.

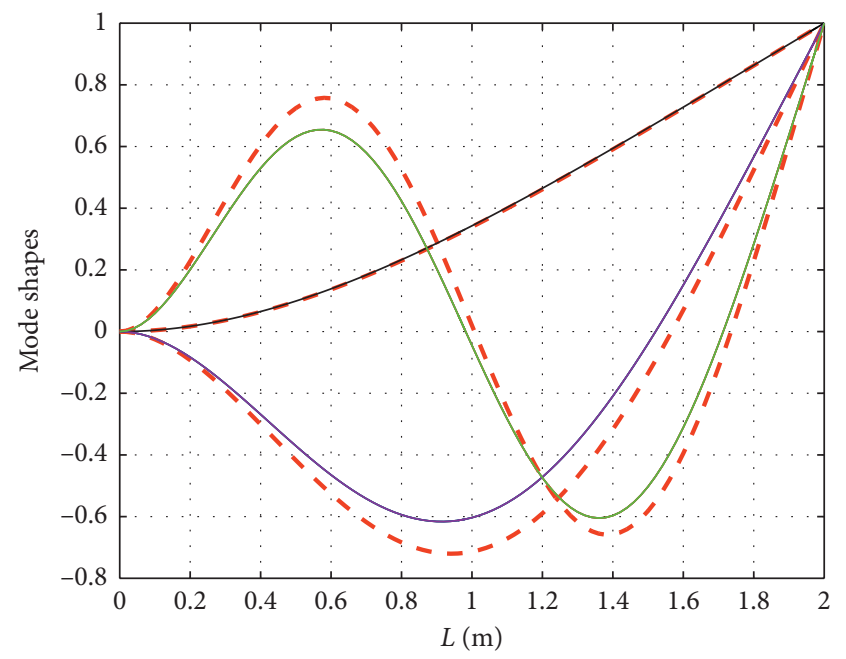

FIgURE 2: First three mode shapes normalized to the largest component equal to unity. Constant mass density (solid lines). Nonconstant mass density (dashed lines).

the modes, and the modal masses are equal for all the modes and so is the length of the mode shapes.

In order to study the convergence of the equations proposed in this paper, the finite element model was discretized with different number of linear bending finite elements (Figure 1) ranging from 2 to 100. Moreover, both consistent and lumped mass matrices were considered in the simulations.

The modal masses estimated with equation (13) for the first three modes using different number of elements are presented in Figure 3. It can be observed that the estimated modal masses converge very fast to the analytical solution when a consistent mass matrix is used, the error being less than $1 \%$ for all the modes when the model is discretized with 6 elements. When a lumped mass matrix is considered, the convergence is achieved with more elements (error less than $1 \%$ with 9, 23, and 36 elements for the first, second, and third modes, respectively). Due to the fact that the mass density is constant, the ratio $m / L^{2}$ is constant for all the modes, and the same conclusions can be inferred for the length of the mode shapes (Figure 4).

Table 2 presents the results obtained with a reduced model using SEREP when only three DOFs are considered. As expected from the theory formulated in Section 6, the modal masses and the length of the mode shapes of the reduced model are exactly the same as those of the full model, independent of the number and the distribution of the active DOFs. The reduced mass and volume matrices are also shown in Table 2.

6.2. A Cantilever Beam with Nonconstant Density. Simulations were also performed on the same cantilever beam but with nonconstant mass density. The geometric and material properties of the cross section are shown in Figure 5.

The analytical mode shapes corresponding to the first three modes and normalized to the largest component equal to unity are shown in Figure 2 (dashed lines). It can be observed that the first mode shape is very similar to that corresponding to the constant mass density case, but significant differences can be seen in the second and third modes.

The analytical modal masses and the squared length of the mode shapes are presented in Table 1 . As the mass density is not constant, equation (25) does not hold, and an apparent mass can be calculated for each mode (see Table 1).

The finite element model was discretized with different number of linear bending elements ranging from 4 to 100 . Both consistent and lumped mass matrices were considered in the simulations.

The modal masses estimated with equation (14) using different number of elements are presented in Figure 6. It can be observed that, again, the estimated modal masses converge very fast to the analytical solution when a consistent mass matrix is used, the error being less than $1 \%$ for all the modes with 7 elements. When a lumped mass matrix is considered, the convergence is achieved with more elements (error less than $1 \%$ with 12,24 , and 36 elements for the first, second, and third modes, respectively).

With respect to the length of the mode shapes (Figure 7), the same speed of convergence as that observed for the constant mass density case was obtained for the nonconstant density case (error less than 1\% with 6 elements and consistent mass matrix).

6.3. A Rigid Block on Springs. In order to illustrate how the equations proposed in this paper work on discrete systems modelled with lumped masses and lumped inertias, the modal masses and mode shape lengths of a rigid block on two springs (see Tables 3-5) vibrating in the $x-y$ plane (bouncing mode and pitch mode) have been calculated. We are illustrating the equations corresponding to both the continuous and the discrete case. 
TABle 1: Modal masses, effective masses, and length of mode shapes for the cantilever beams.

\begin{tabular}{|c|c|c|}
\hline & \multicolumn{2}{|c|}{ Model } \\
\hline & Constant density (Figure 1) & Nonconstant density (Figure 5) \\
\hline Total mass (kg) & 16 & 16 \\
\hline Apparent mass (kg) & Equal to total mass, $M_{a 1}=M_{a 2}=M_{a 3}=16$ & $\begin{array}{l}M_{a 1}=14.10 \\
M_{a 2}=15.93 \\
M_{a 3}=16.06\end{array}$ \\
\hline Squared length of mode shapes & $L_{1}^{2}=L_{2}^{2}=L_{3}^{2}=0.25$ & $\begin{array}{l}L_{1}^{2}=0.2517 \\
L_{2}^{2}=0.2049 \\
L_{3}^{2}=0.2063\end{array}$ \\
\hline Modal mass (kg) & $m_{1}=m_{2}=m_{3}=4$ & $\begin{array}{l}m_{1}=3.549 \\
m_{2}=3.265 \\
m_{3}=3.313\end{array}$ \\
\hline
\end{tabular}
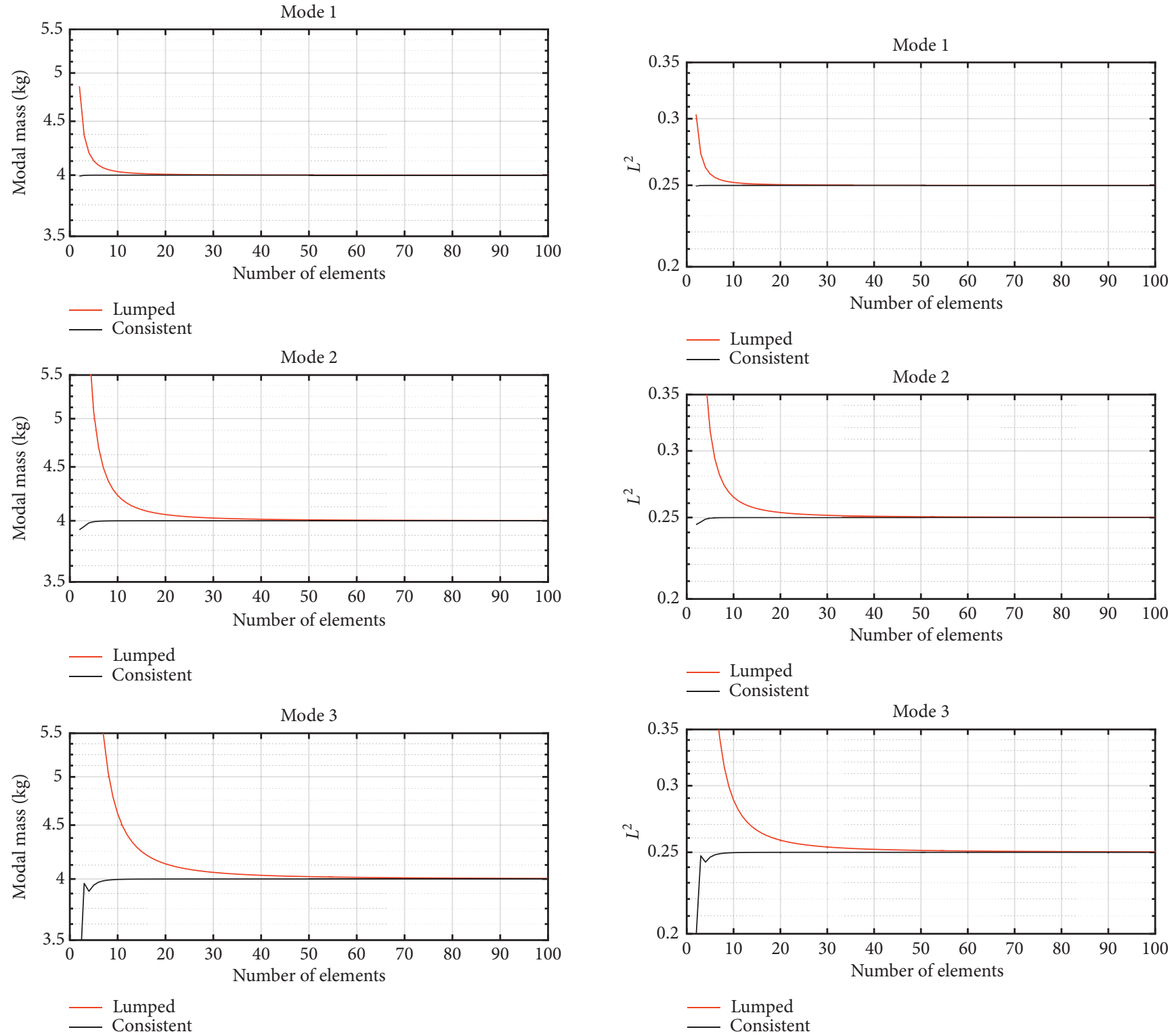

FIgURE 3: Convergence of the modal masses for the first three modes of the cantilever beam with constant mass density.

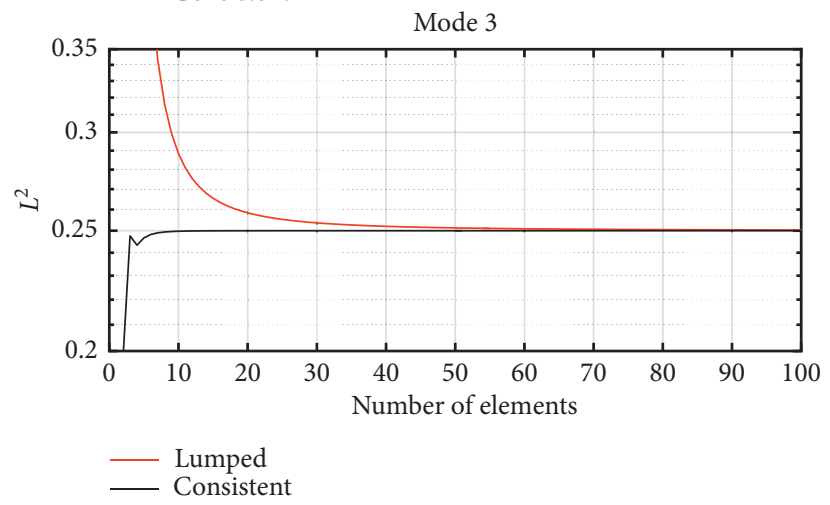

FIgURE 4: Convergence of the length of the mode shapes for the first three modes of the cantilever beam with constant mass density. 
TABle 2: Results obtained with a reduced model using SEREP [45].

Active DOFs

$[33,65,99]$

$[49,75,99]$

Mass matrix (kg)

$\left[\begin{array}{ccc}18681 & -12941 & 4336 \\ -12941 & 8972 & -3009 \\ 4336 & -3009 & 1017\end{array}\right]$

$\left[\begin{array}{rrr}7083 & -8616 & 3735\end{array}\right.$

$\begin{array}{lll}-8616 & 10493 & -4554\end{array}$

$\begin{array}{lll}3735 & -4554 & 1985\end{array}$

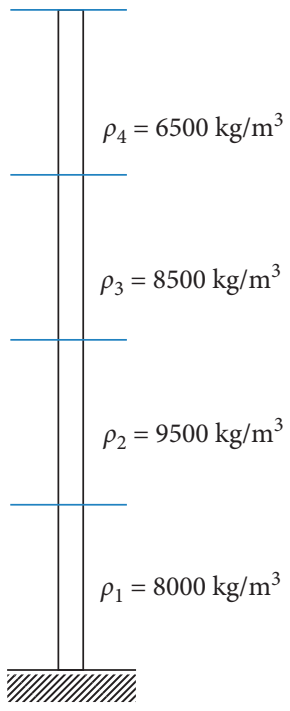

Figure 5: Cantilever beam with nonconstant mass density.

The block has constant density $\rho$, dimensions $a \times b \times c$, total mass $\mathbf{M}=\rho a b c$, total volume $V=\mathbf{M} / \rho$, and inertia $J=$ $\mathbf{M} / 12\left(a^{2}+b^{2}\right)$ with respect to the center of gravity of the block.

If we consider the block as a continuous system, the analytical expressions of the mode shapes in both directions, $x$ and $y$, are needed (see Table 3 ). The mode shapes have been normalized to the largest vertical component equal to unity. The modal masses and the length of the mode shapes calculated with equations (16) and (18) are presented in Table 3.

If the system is modelled with two translational DOFs (see Table 4), the mass matrix, the volume matrices, and the mode shapes are presented in Table 4 . The modal masses and the mode shape lengths calculated with equations (37) and (38), respectively, are shown in Table 4.

The results obtained with the same system modelled with one translation and one rotational DOF are shown in Table 5.
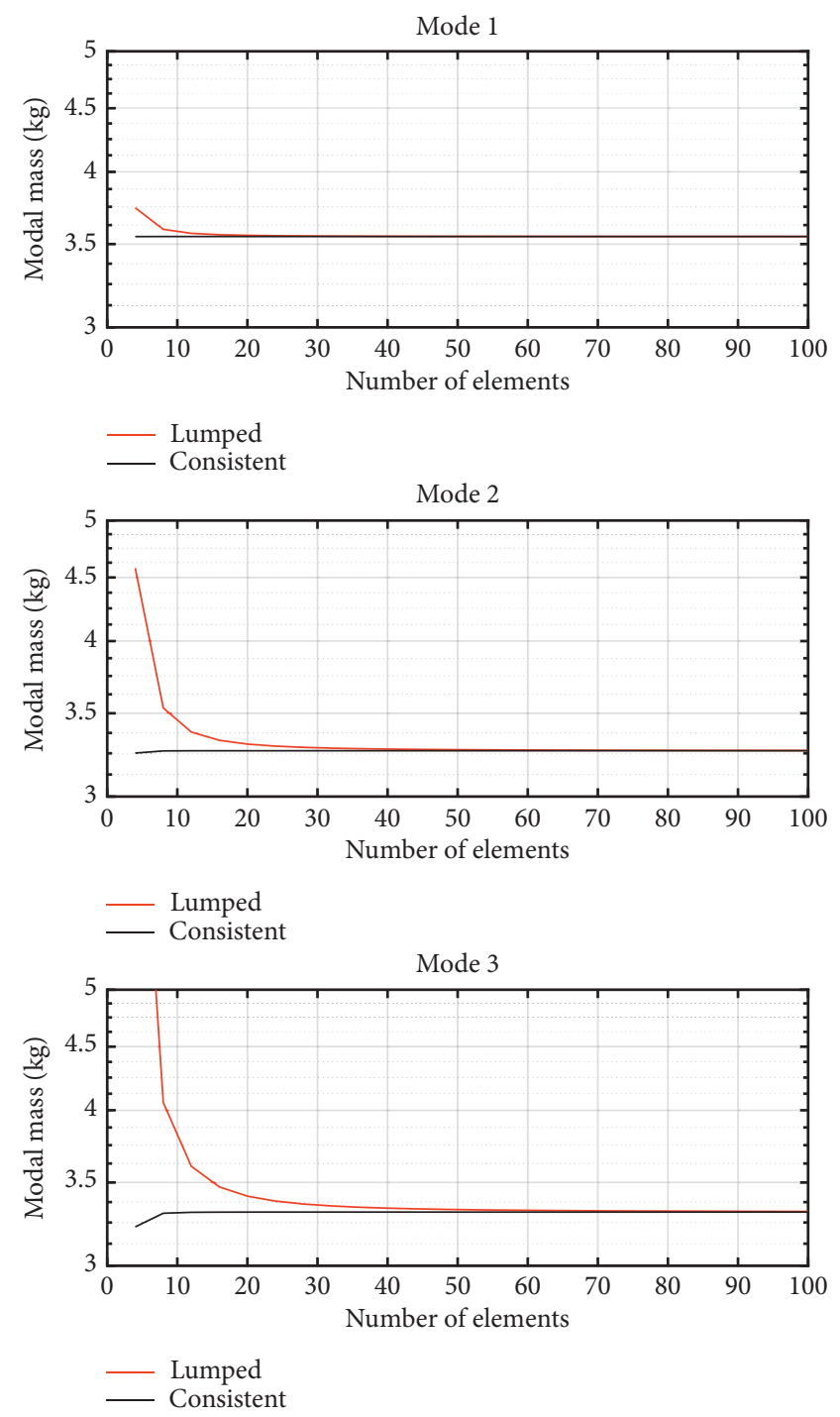

Figure 6: Convergence of the modal masses for the first three modes of the cantilever beam with nonconstant mass density. 

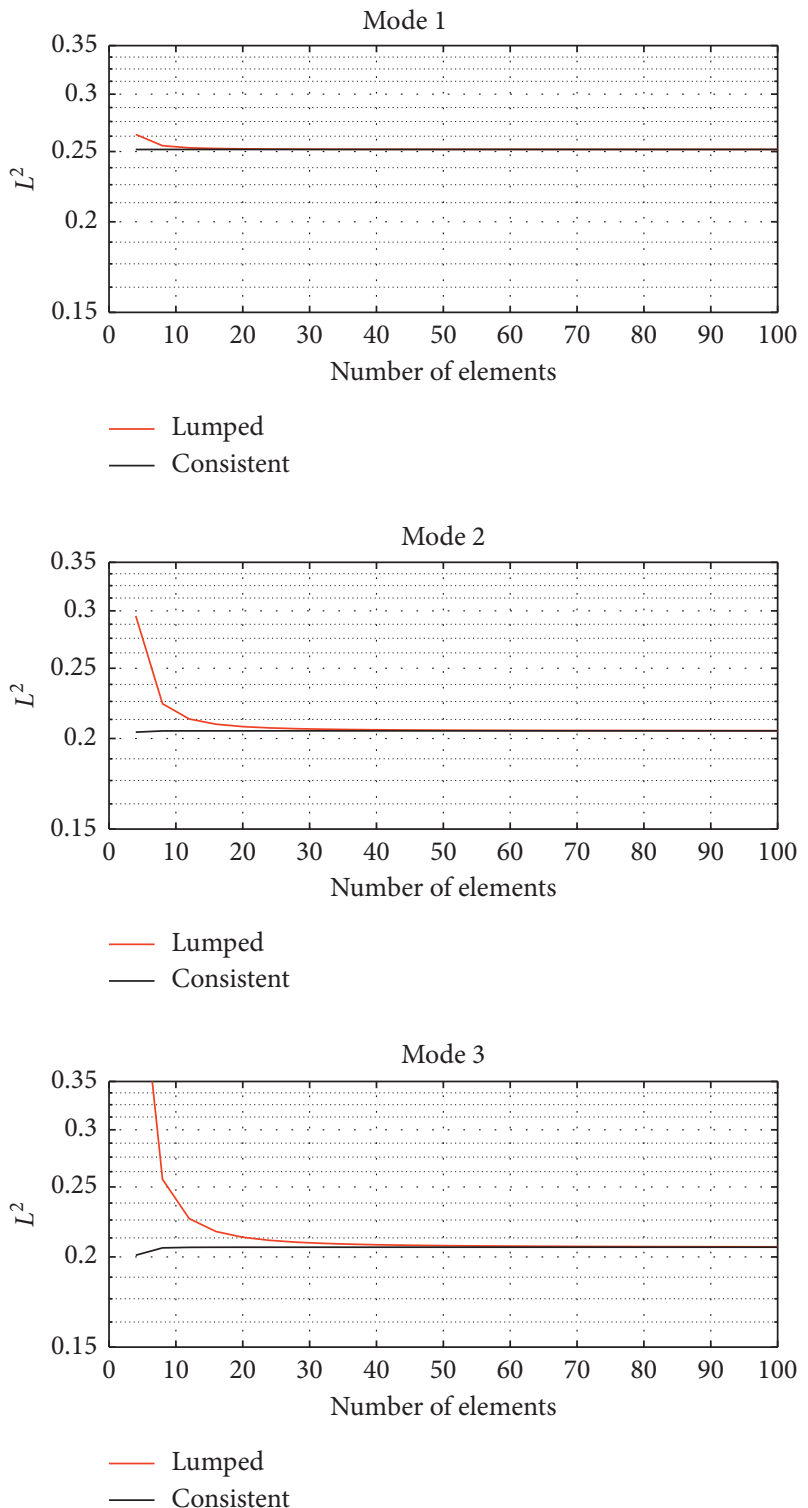

FIGURE 7: Convergence of the length of the mode shapes for the first three modes of the cantilever beam with nonconstant mass density.

In can be seen from Tables 3-5 that the obtained values of the mode shape lengths and the modal masses are the same using different models. Moreover, as the density is constant, the modal mass is equal to the product between the total mass of the structure and the squared length. This example demonstrates that the concepts and equations formulated in this paper can also be easily extended to discrete systems modelled with lumped masses and/or lumped inertias.

\section{Discussion}

Considering a certain body, first, one has to decide which parts of the body have constant mass density. Let us first assume that we can identify a number of different bodies with the volumes $V_{n}$, where this is the case. Then, for each of these partial bodies, the corresponding modal mass and modal length are calculated according equations (9) and (37):

$$
\begin{aligned}
& m_{n}=\psi_{\mathbf{n}}^{\mathrm{T}} \mathbf{M}_{\mathbf{n}} \psi_{\mathbf{n}}, \\
& L_{n}^{2}=\frac{1}{V_{n}} \psi_{\mathbf{n}}^{\mathrm{T}} \mathbf{V}_{\mathbf{n}} \psi_{\mathbf{n}},
\end{aligned}
$$

where $\psi_{n}$ is the part of the mode shape $\psi$ corresponding to the considered partial body $V_{n}, m_{n}$ and $L_{n}^{2}$ are the contributions to the modal mass and the mode shape length in the body, and $V_{n}$ is the volume of the body. $\mathbf{M}_{\mathbf{n}}$ is the mass matrix, and $\mathbf{V}_{\mathbf{n}}$ is the volume matrix of the considered partial body, respectively, where $\mathbf{V}_{\mathbf{n}}$ is found from equation (44). This means that we can calculate separately the modal mass $m_{n}$ and the length $L_{n}^{2}$ of each partial body $V_{n}$ (for example, in a finite element model, we can calculate the modal mass and the length of each of the finite elements of the model) and then calculate the total modal mass and the total length as the sum of the contributions of each body. 
TABle 3: A rigid block on springs. Continuous formulas.

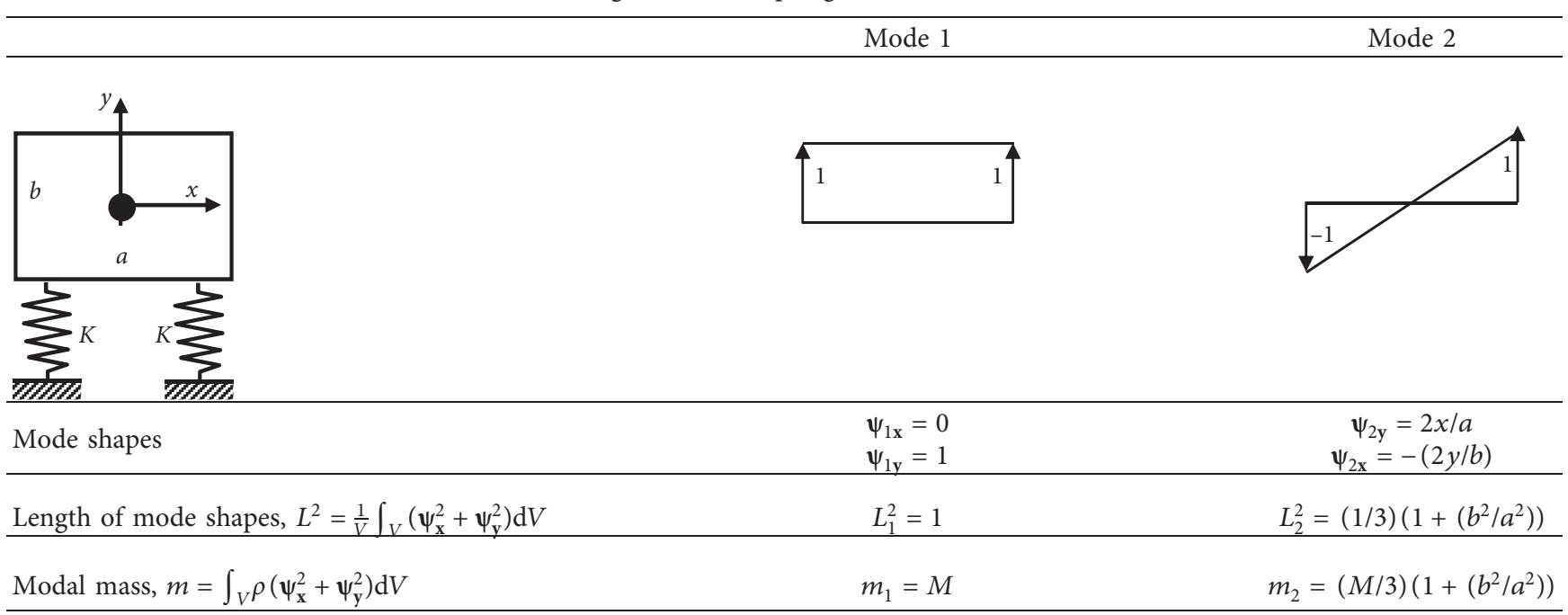

TABle 4: A rigid block on springs. Discretization with two translational DOFs.

Mode 1
Mode 2
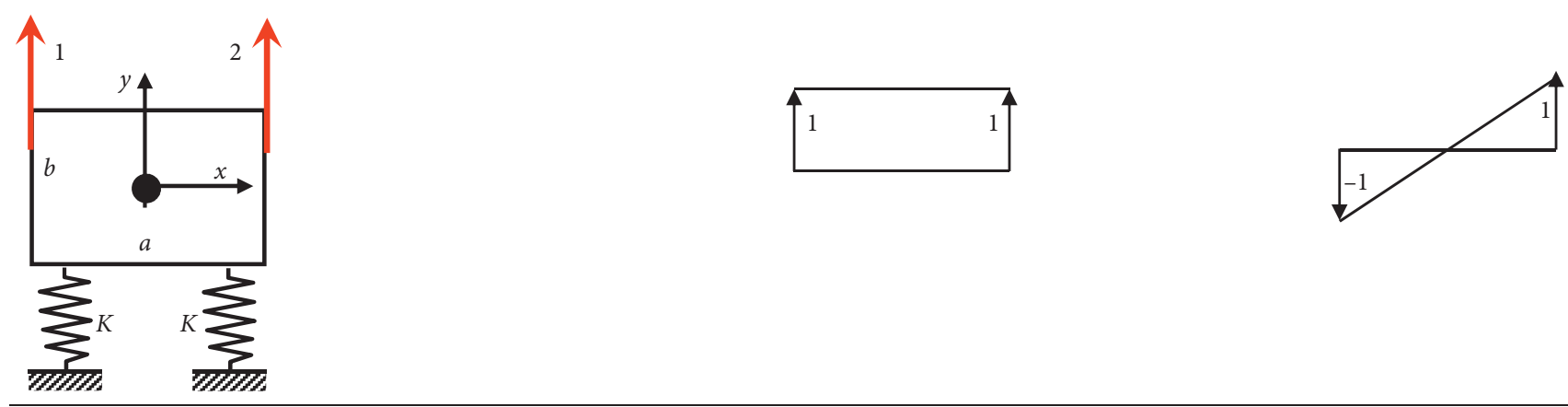

Mode shapes

$\psi_{1}=\left\{\begin{array}{l}1 \\ 1\end{array}\right\}$

$\psi_{2}=\left\{\begin{array}{c}-1 \\ 1\end{array}\right\}$

Mass matrix $\mathbf{M}$

$\left[\begin{array}{cc}(M / 4)+\left(J / a^{2}\right) & (M / 4)-\left(J / a^{2}\right) \\ (M / 4)-\left(J / a^{2}\right) & (M / 4)+\left(J / a^{2}\right)\end{array}\right]$

Volume matrix $\mathbf{V}$

$\left[\begin{array}{ll}(V / 4)+(V / 12)\left(1+\left(b^{2} / a^{2}\right)\right) & (V / 4)-(V / 12)\left(1+\left(b^{2} / a^{2}\right)\right) \\ (V / 4)-(V / 12)\left(1+\left(b^{2} / a^{2}\right)\right) & (V / 4)+(V / 12)\left(1+\left(b^{2} / a^{2}\right)\right)\end{array}\right]$

Length of mode shapes, $L^{2}=(1 / V) \psi^{\mathrm{T}} \mathbf{V} \psi$

Modal mass, $m=\psi^{\mathrm{T}} \mathbf{M} \psi$

$L_{1}^{2}=1$

$L_{2}^{2}=(1 / 3)\left(1+\left(b^{2} / a^{2}\right)\right)$

$m_{1}=M$

$m_{2}=(M / 3)\left(1+\left(b^{2} / a^{2}\right)\right)$

The total mass and the total volume of each partial body are then

$$
m_{n}=\mathbf{M}_{\mathbf{n}} \mathbf{L}_{\mathbf{n}}^{2}
$$

$$
\begin{aligned}
M_{n} & =\psi_{\mathrm{U}}^{\mathrm{T}} \mathbf{M}_{\mathbf{n}} \psi_{\mathrm{U}}, \\
V_{n} & =\psi_{\mathrm{U}}^{\mathrm{T}} \mathbf{V}_{\mathbf{n}} \psi_{\mathrm{U}},
\end{aligned}
$$

where $\psi_{\mathrm{U}}$ is a unitary translation. For each partial body, the so-calculated quantities should satisfy equation (47):

for all modes. If this is not the case, the apparent total masses, as introduced in equation (39), are not equal to the total mass; then, it means that the assumption of constant mass density inside the considered partial body is wrong, and the body should be subdivided into smaller parts. However, if equation (65) holds for all partial bodies and all 
TABLE 5: A rigid block on springs. Discretization with one translation and one rotation.

Mode shapes

Length of mode shapes, $L^{2}=(1 / V) \psi^{\mathrm{T}} \mathbf{V} \psi$

$L_{1}^{2}=1$

$L_{2}^{2}=(1 / 3)\left(1+\left(b^{2} / a^{2}\right)\right)$

Modal mass, $m=\psi^{\mathrm{T}} \mathbf{M} \psi$

$m_{1}=M$

$m_{2}=(M / 3)\left(1+\left(b^{2} / a^{2}\right)\right)$

modes, then we can conclude the final results for the total body:

$$
m=\sum_{n} m_{n}, M=\sum_{n} M_{n}, V=\sum_{n} V_{n}, L^{2}=\sum_{n} L_{n}^{2} .
$$

If we are not working with well-defined parts each having its own constant mass density but rather a density that is varying smoothly over the body, then it might be more convenient to use all elements as partial bodies. In this case, we can follow the same procedure as mentioned above, but where the volume matrix $\mathbf{V}_{\mathbf{n}}$ is now simply found from equation (49).

The final question still remains-how should we choose the scaling? The answer is that it does not matter because whatever scaling we use, a linear relation exists between squared length and the modal mass given by equation (39), and we can accurately calculate the relation for any of the considered modes.

At this point, it is worth noticing that we can in a way forget about the dead mass problem because any influence is removed using equation (39). However, of course, if the length contributions from all modes are zero for a certain partial body, then it might be useful to remove that body from the description/modelling of the problem.

Finally, coming back to the initial requirements mentioned in Section 2, we can conclude that most of them have been fulfilled with the present proposal. Both modal mass and mode shape length have now reasonable units and a well-defined meaning. Also, it is easy for anybody to achieve the same result no matter what scaling has been used because the final answer is not the modal mass, and the final answer is all quantities need to be defined by equation (49). However, one can say that since the DOF scaling $\max \left|\psi_{D}\right|=1$ is more meaningful than the length scaling and because the modal masses using DOF scaling clearly illustrate the amount of mass moving under the considered mode shape, it might, in many cases, be more meaningful to use this scaling than any other possible scaling.

\section{Conclusions}

The concept of length of a continuous mode shape has been defined in this paper. The new definition depends on the mode shape and how the volume is distributed in the structure. Then, the concept of length has been extended to discrete systems by introducing the concept of a volume matrix. This length definition has the same unit as the mode shape, but it does not coincide with the length of a vector in an Euclidean space that is normally used in linear algebra. We omit the definition from linear algebra because in that case, the length depends on the number of components of the vector.

A new and better definition of the modal mass, which is physically meaningful and does not depend on the number of DOFs of a discrete model, has been formulated. It is demonstrated that if the mass density of the system is constant, then the modal mass is always equal to the product between the total mass of the structure and the squared length.

If the mass density is not constant, the concept of apparent mass is proposed. This apparent mass is different for 
each mode and depends on the mode shape and how the mass is distributed in the structure. In these cases, the modal mass is always equal to the product between the apparent mass and the length squared.

If a model is reduced with the SEREP reduction technique, it has been demonstrated that the modal mass and the length of the mode shapes are invariant to SEREP, i.e., the modal mass and the modal length are the same in all reduced systems and equal to the modal mass and length of the full model.

In constant mass density systems, when mode shapes are normalized to the length, the modal mass is always equal to the total mass of the considered system. The drawback is that this modal mass does not give us any information about how much mass is actually moving in different modes. For example, local and global modes have the same modal mass, in spite of the fact that global modes are moving much more mass. In nonconstant mass density systems, the modal mass is different for each mode and equal to an apparent mass.

When mode shapes are normalized to the largest component equal to unity, the modal mass is a measure of how much mass is moving in the considered mode. The drawback is that we need to scale the maximum component equal to unity, and in a model with only a few DOFs, where the DOF with the largest component is not measured, it is not easy to find the right scaling because we have to find the largest component in the converged model.

\section{Data Availability}

The data supporting the results reported in this paper are available from the corresponding author upon request.

\section{Conflicts of Interest}

The authors declare that they have no conflicts of interest in this work.

\section{Acknowledgments}

The financial support provided by the Banco Santander through the Mobility Grants for Researchers and Professors of the University of Oviedo is gratefully appreciated. The authors acknowledge the funding received from the Centre for Oil and Gas-DTU/Danish Hydrocarbon Research and Technology Centre (DHRTC).

\section{References}

[1] D. J. Ewins, Modal Testing: Theory, Practice and Application, Research Studies Press LTD, London, UK, 2nd edition, 2000.

[2] N. M. M. Maia and J. M. Montalvao e Silva, Theoretical and Experimental Modal Analysis, Research Studies Press LTD, London, UK, 2003.

[3] W. Heylen, S. Lammens, and P. Sas, Modal Analysis Theory and Testing, Katholieke Universiteit Leuven, Leuven, Belgium, 2007.

[4] R. Brincker and C. Ventura, Introduction to Operational Modal Analysis, John Wiley \& Sons, Chichester, England, 2015.
[5] R. W. Clough and J. Penzien, Dynamics of Structures, McGraw-Hill, New York, NY, USA, 2nd edition, 1993.

[6] A. K. Chopra, Dynamics of Structures, Prentice-Hall, Upper Saddle River, NJ, USA, 1995.

[7] M. Paz and W. Leigh, Structural Dynamics Theory and Computation, Kluwer Academic Publishers, Boston, MA, USA, 1997.

[8] S. S. Rao, Vibration of Continuous Systems, John Wiley \& Sons, Hoboken, NJ, USA, 2007.

[9] A. W. Leissa and M. S. Qatu, Vibration Continuous Systems, McGraw-Hill, New York, NY, USA, 2011.

[10] M. I. Friswell and M. I. Mottershead, Finite Element Model Updating in Structural Dynamics, Kluwer Academic Publishers, London, UK, 1999.

[11] M. H. Richardson, Modal Mass, Stiffness and Damping, Vibrant Technology Inc., Centennial, CO, USA, 2000.

[12] P. Rochus, J. M. Defise, J. Y. Plesseria, F. Hault, and G. Janssen, "Effective modal parameters to evaluate structural stresses," in Proceedings of the European Conference on Spacecraft Structures, Materials and Mechanical Testing, Braunschweig, Germany, November 1998.

[13] W. T. Thomson, Theory of Vibration with Applications, Prentice-Hall, Upper Saddle River, NJ, USA, 5th edition, 2013.

[14] R. M. Bamford, B. K. Wada, and W. H. Gayman, "Equivalent spring- miss system for normal modes," NASA Technical Report, NASA, Washington, DC, USA, 1971.

[15] R. L. Mayes, T. F. Schoenherr, J. Blecke, and D. P. Rohe, "Efficient method of measuring effective mass of a system," in Conference Proceedings of the Society for Experimental Mechanics Series, Springer, Berlin, Germany, 2014.

[16] R. Sedaghati, Y. Soucy, and N. Etienne, "Experimental estimation of effective mass for structural dynamics and vibration applications," in Proceedings of the 21st Intern ational Modal Analysis Conference (IMAC XXI), Kissimmee, FL, USA, February 2003.

[17] Ç. Hizal and G. Turan, "Importance of static correction and damping in the analysis of a cable-stayed bridge subjected to displacement loading," Journal of Bridge Engineering, vol. 22, no. 6, Article ID 04017009, 2017.

[18] Ç. Hızal and G. Turan, "Effect of seismic wave velocity on the dynamic response of multi-story structures on elastic foundation," Soil Dynamics and Earthquake Engineering, vol. 113, pp. 432-441, 2018.

[19] S. Zivanovic, A. Pavic, and P. Reynolds, "Modal testing and FE model tuning of a lively footbridge structure," Engineering Structures, vol. 28, no. 6, pp. 857-868, 2006.

[20] M. S. Allen and M. W. Sracic, "Mass normalized mode shapes using impact excitation and continuous-scan laser Doppler vibrometry," in Proceedings of the 8th International Conference on Vibration Measurements by Laser Techniques: Advances and Applications, Ancona, Italy, June 2008.

[21] R. Brincker and P. Andersen, "A way of getting scaled mode shapes in output only modal analysis," in Proceedings of the International Modal Analysis Conference (IMAC XXI), Orlando, FL, USA, February 2003.

[22] M. López-Aenlle, P. Fernández, R. Brincker, and A. Fernández-Canteli, "Erratum to "scaling-factor estimation using an optimized mass-change strategy"” Mechanical Systems and Signal Processing, vol. 24, no. 8, pp. 3061-3074, 2010.

[23] M. López-Aenlle, R. Brincker, P. Fernández, and A. Fernández-Canteli, "On exact and approximated formulations for scaling mode shapes in operational modal analysis by mass and stiffness change," Journal of Sound and Vibration, vol. 331, no. 3, pp. 622-637, 2012. 
[24] D. Bernal, "A receptance based formulation for modal scaling using mass perturbations," Mechanical Systems and Signal Processing, vol. 25, no. 2, pp. 621-629, 2011.

[25] D. Bernal, "Modal scaling from known mass perturbations," Journal of Engineering Mechanics, vol. 130, no. 9, pp. 10831088, 2004.

[26] M. L. Aenlle and R. Brincker, "Modal scaling in operational modal analysis using a finite element model," International Journal of Mechanical Sciences, vol. 76, pp. 86-101, 2013.

[27] B. Schwarz and M. Richardson, "Using FEA modes to scale experimental mode shapes," in Proceedings of the International Modal Analysis Conference, Scotts Valley, CA, USA, January 2006.

[28] J. M. W. Brownjohn and A. Pavic, "Experimental methods for estimating modal mass in footbridges using human-induced dynamic excitation," Engineering Structures, vol. 29, no. 11, pp. 2833-2843, 2007.

[29] J. M. W. Brownjohn, A. Raby, J. Bassitt et al., "Experimental modal analysis of British rock lighthouses," Marine Structures, vol. 62, pp. 1-22, 2018.

[30] A. Brandt, M. Berardengo, M. Berardengo, S. Manzoni, M. Vanali, and A. Cigada, "Global scaling of operational modal analysis modes with the OMAH method," Mechanical Systems and Signal Processing, vol. 117, pp. 52-64, 2019.

[31] J. Cara, "Computing the modal mass from the state space model in combined experimental-operational modal analysis," Journal of Sound and Vibration, vol. 370, pp. 94-110, 2016.

[32] P. Guillaume, R. De Troyer, C. Devriendt, and G. De Sitter, "OMAX-a combined experimental-operational modal analysis approach," Proceedings of the International Conference on Noise and Vibration Engineering, ISMA, vol. 1, pp. 2985-2996, 2006.

[33] J.-S. Hwang, S. H. Lee, S.-H. Lee, and A. Kareem, "Estimation of modal masses of a structure with a mass-type damping device," Engineering Structures, vol. 99, pp. 149-156, 2015.

[34] U. Fullekrug, "Determination of effective masses and modal masses from base-driven tests," in Proceedings of the International Modal Analysis Conference (IMAC XIV), Dearborn, MI, USA, February 1996.

[35] J. Ormondroyd and J. P. Den Hartog, "The theory of dynamic vibration absorber," Transactions of the ASME, vol. APM-507, pp. 9-22, 1928.

[36] P. H. Wirsching and J. T. P. Yao, "Safety design concepts for seismic structures," Computers \& Structures, vol. 3, no. 4, pp. 809-826, 1973.

[37] S. E. Gaur, T. Höbbel, V. A. Matsagar, and K. Thiele, “Tuned mass dampers in wind response control of wind turbine with soil-structure interaction," Soil Dynamics and Earthquake Engineering, vol. 132, 2020.

[38] K. Moon, "The structural design of tall and special buildings," The Structural Design of Tall Special Buildings, vol. 29, no. 9, pp. 347-366, 2019.

[39] S. Elias, V. Matsagar, and T. K. Datta, "Dynamic response control of a wind-excited tall building with distributed multiple tuned mass dampers," International Journal of Structural Stability and Dynamics, vol. 19, 2019.

[40] J.-S. Hwang, H. Kim, and J. Kim, "Estimation of the modal mass of a structure with a tuned-mass damper using $\mathrm{H}$-infinity optimal model reduction," Engineering Structures, vol. 28, no. 1, pp. 34-42, 2006.

[41] S. Elías, "Effect of SSI on vibration control of structures with tuned vibration absorbers," Shock and Vibration, vol. 2019, Article ID 7463031, 12 pages, 2019.
[42] Moon, "Performance of tuned mass damper/double skin façade damping interaction systems," in Proceedings of the 7th International Conference on Modern Research in Civil Engineering, Architectural \& Urban Development, Munich, Germany, October 2018.

[43] S. A. Dianat and E. Saber, Advanced Linear Algebra for Engineers Wiht MATLAB, CRC Press, New York, NY, USA, 2009.

[44] C. Q. Howard, "Modal mass of clamped beams and clamped plates," Journal of Sound and Vibration, vol. 301, no. 1-2, pp. 410-414, 2007.

[45] J. C. O'Callahan, P. Avitabile, and R. Riemer, "System equivalent reduction expansion process," in Proceedings of the 7th international Modal Analysis Conference (IMAC), pp. 29-37, Las Vegas, NV, USA, January 1989. 